\title{
Complicación posfibrinólisis como suelta embólica de trombo intracavitario no conocido
}

\author{
Juncal Sánchez-Arguiano', Maite Arlabán-Carpintero', Enrique Chicote-Álvarez', María Ángeles Ballesteros-Sanz', \\ Manuel Gayol-Fernández ${ }^{2}$ \\ 'Servicio de Medicina Intensiva. Hospital Universitario Marqués de Valdecilla. Santander (Cantabria). España \\ ${ }^{2}$ Atención Primaria. Servicio de Salud del Principado de Asturias. España
}

Recibido: 07/07/2018

Aceptado: 26/10/2018

En línea: 31/12/2018

Citar como: Sánchez-Arguiano J, Arlabán-Carpintero M, Chicote-Álvarez E, Ballesteros-Sanz MA, Gayol-Fernández M. Complicación posfibrinólisis como suelta embólica de trombo intracavitario no conocido. Rev Esp Casos Clin Med Intern (RECCMI). 2018 (Dic); 3(3): 107-109. doi: 10.32818/reccmi.a3n3a2.

Autor para correspondencia: Juncal Sánchez-Arguiano. mjuncalsar@gmail.com

\section{Palabras clave \\ $\triangleright$ Trombo móvil cardíaco \\ $\triangleright$ Trombólisis \\ $\triangleright$ Ictus múltiple}

\begin{abstract}
Resumen
La terapia fibrinolítica es el pilar del tratamiento del accidente cerebrovascular isquémico. Las complicaciones embólicas como consecuencia del tratamiento fibrinolítico están descritas en casos escasos en la literatura, y se cree que puedan deberse a la desintegración de un trombo ya formado previamente. Se presenta el caso de una paciente de 63 años que ingresa por accidente cerebrovascular agudo isquémico de la arteria cerebral media izquierda, que se somete a fibrinólisis y que sufre posteriormente infartos múltiples en distintos territorios vasculares como consecuencia de una liberación de fragmentos de un trombo intraventricular no conocido. Se establece como conclusión que ante un deterioro neurológico temprano tras la administración de fibrinolítico, si además asocia clínica correspondiente a otro territorio vascular distinto del inicialmente afectado, deberíamos pensar en esta entidad.
\end{abstract}

\begin{tabular}{l} 
Keywords \\
\hline$\triangleright$ Mobile cardiac thrombus \\
$\triangleright$ Thrombolysis \\
$\triangleright$ Multiple ictus
\end{tabular}

\section{Puntos destacados}

$\triangleright$ Se presenta el caso de una suelta embólica de un trombo intracavitario no conocido, posfibrinólisis, con el desarrollo de múltiples áreas isquémicas cerebrales.

$\triangleright$ Dicha entidad se debe barajar como diagnóstico diferencial ante un deterioro neurológico temprano tras terapia fibrinolítica.

\section{Introducción}

El accidente cerebrovascular agudo (ACVA) isquémico es una de las primeras causas de muerte en nuestro medio, siendo la terapia fibrinolítica el pilar del tratamiento precoz, idealmente inferior a 4,5 horas', aprobada en España desde 2003. Las posibles complicaciones embólicas o el ictus recurrente tras la terapia fibrinolítica en el ACVA isquémico son incidentes anecdóticos, que se piensa que puedan ser debidos a la desintegración de un trombo previamente ya formado en otra localización.

\section{Caso clínico}

Presentamos el caso de una mujer con múltiples embolismos tras la realización de una fibrinólisis terapéutica de un ACVA de perfil cardioembólico en la región de la arteria cerebral media izquierda (ACMI). Se trata de una mujer de 63 años, con múltiples factores de riesgo cardiovascular (hipertensión, dislipemia y diabetes mellitus tipo 2); en tratamiento con enalapril, metformina y vidagliptina; y portadora de marcapasos por enfermedad del seno; que ingresa por hemiparesia derecha y afasia motora. Se activa protocolo Código ICTUS y se realiza una tomografía axial computarizada (TAC) basal, así como TAC de 
perfusión cerebral. Dichas pruebas mostraban defectos de repleción en ramas distales de ACMI, diagnosticándose de ictus isquémico agudo de ACMI de perfil cardioembólico, con menos de 3 horas de evolución. Se decide inicio de terapia fibrinolítica intravenosa (alteplasa), según protocolo habitual del centro, constatándose una mejoría en los minutos posteriores, con presencia de parálisis facial central derecha moderada, disartria y extinción visual.

Aproximadamente 45 minutos después del procedimiento, sufre un deterioro súbito del nivel de consciencia y crisis tónico-clónicas generalizadas. Se realiza TAC basal que descarta complicaciones hemorrágicas posfibrinólisis y, ante la persistencia de un nivel de consciencia bajo (escala de coma de Glasgow [GCS] 5), se decide intubación orotraqueal y traslado a Cuidados Intensivos, diagnosticándose como probable crisis tónico-clónica tras ACVA. Durante su estancia en la unidad, la paciente permanece estable sin necesidad de infusión de aminas, las analíticas de rutina realizadas (bioquímica, hematimetría y coagulación) se encontraban en el rango de la normalidad, y se mantiene con ritmo de marcapasos a 60 latidos por minuto.

En las 10 horas posteriores, se retira la sedoanalgesia para nueva exploración neurológica, constatándose un valor de GCS de 3, por lo que se realiza nueva TAC craneal que muestra áreas hipodensas en región frontoparietal derecha así como en frontoinsular y parietal alta izquierdas compatibles con isquemia aguda (Figura 1). La paciente evoluciona de manera desfavorable en las horas posteriores hasta la muerte encefálica. Se realiza ecocardiografía transesofágica (Figura 2) en la que se evidencia trombo digitiforme móvil en la cavidad ventricular izquierda anclada a zona acinética apical, sin otras alteraciones ecocardiográficas destacables (cavidades no dilatadas y sin valvulopatías reseñables). Finalmente, es considerada como donante multiórgano, evidenciándose durante la extracción orgánica una isquemia mesentérica masiva.

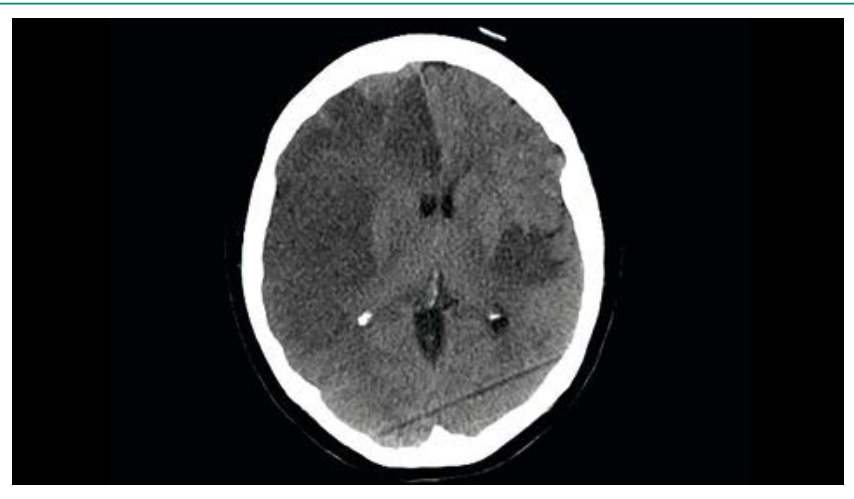

Figura 1. Corte transversal de TAC craneal que muestra áreas isquémicas en al menos dos territorios vasculares cerebrales distintos
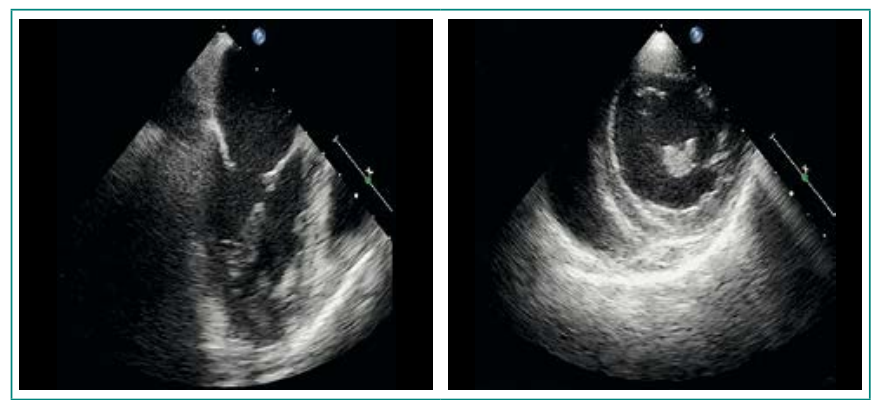

Figura 2. Imágenes de ecocardiografía transesofágica que muestran el trombo móvil y pediculado. En eje longitudinal de dos cámaras (izquierda), trombo anclado a ápex. En eje corto de dos cámaras (derecha), trombo pediculado a la altura de músculos papilares

\section{Discusión}

Los casos descritos en la bibliografía como complicaciones de ACVA posfibrinólisis hacen referencia, en su mayoría, al desarrollo de hemorragias o angioedema orolingual ${ }^{2}$. Las complicaciones embólicas como complicación del propio tratamiento del ictus se registran de forma escasa en la literatura ${ }^{3,4} y$ pueden ser como complicaciones trombóticas a otros niveles ${ }^{5}$, como en el caso que se describe, en arterias del polígono de Willis ${ }^{3,4}$, siendo más frecuentes los desprendimientos de trombos intracavitarios que produzcan ACV isquémicos, bien espontáneos ${ }^{6}$, bien postratamiento fibrinolítico de un infarto ${ }^{4,7}$ que pudo producir una desestabilización del trombo preexistente con dicho tratamiento. La existencia de un trombo previo constituye un factor de riesgo esencial para el desarrollo de la propia entidad ${ }^{7}$. Además, con respecto a la etiología de las otras entidades, no puede descartarse la preexistencia de un trombo que pudiera haber desaparecido tras el tratamiento fibrinolítico, ya que esto ocurre con cierta frecuencia ${ }^{7}$. Sí se han registrado series de ACV isquémico recurrente tras tratamiento con alteplasa con una tasa del 2,6\%, generalmente asociado a fibrilación auricular ${ }^{3}$. Un deterioro neurológico abrupto, tras o durante la infusión de fibrinolítico, debe hacernos sospechar el desarrollo de un nuevo ACV isquémico ${ }^{8}$ o bien una falta de recanalización de la arteria con progresión de la isquemia o el desarrollo de nuevas complicaciones.

Como factores de riesgo reportados en la bibliografía para el desarrollo de trombos intracardíacos están, entre otros muchos, los siguientes: obesidad, infecciones sistémicas, neoplasias malignas, enfermedades renales, síndrome de distrés respiratorio, enfermedad cardíaca congénita, miocardiopatía dilatada, catéteres intravasculares, enfermedades autoinmunes, estados de hipercoagulabilidad (adquiridos o congénitos), infarto de miocardio (una fracción de eyección inferior al 35\% es uno de los mayores predictores). La mayoría de los casos son pacientes con cardiopatía isquémica ya conocida y/o arritmias ${ }^{3-5}$. En nuestro caso, el único antecedente era la enfermedad del seno.

El tratamiento y pronóstico de estos pacientes es muy variable, debido tanto a las potenciales secuelas como al desconocimiento de esta propia entidad.

En la Unidad de Ictus de nuestro centro se realiza de rutina el estudio cardiológico con ecocardiografía, habitualmente transtorácica, en aquellos pacientes con sospecha de ictus cardioembólico y en todo ictus juvenil, aunque únicamente se realiza de urgencia si hay alta sospecha de endocarditis bacteriana, trombo intracardíaco o zona acinética ventricular (características que, a priori, no cumplía la paciente).

\section{Conclusiones}

Cuando se produce un deterioro neurológico temprano en relación con la administración de fibrinolítico, se debe sospechar fallo en la terapia con recanalización incompleta o no recanalización, o nuevo ACVA hemorrágico o isquémico. En el caso de nuevo ACVA isquémico, debe descartarse que haya sido consecuencia de la fragmentación de un trombo intracardíaco ya presente.

Para nuestro conocimiento, sería conveniente realizar un estudio acerca de esta patología que, aunque infrecuente y raramente reportada, puede tener una alta morbimortalidad. Cabe plantearse si sería necesaria la extensión de los criterios de realización de ecocardiografía urgente. 


\section{Bibliografía}

1. Powers WJ, Derdeyn CP, Biller J, Coffey CS, Hoh BL, Jauch EC, et al. 2015 American Heart Association/American Stroke Association Focused Update of the 2013 guidelines for the early management of patients with acute ischemic stroke regarding endovascular treatment: a guideline for healthcare professionals from the American Heart Association/American Stroke Association. Stroke. 2015; 46: 3020-3035. doi: 10.1161/STR.0000000000000074.

2. Powers WJ, Rabinstein AA, Ackerson T, Adeoye OM, Bambakidis NC, Becker K, Biller J, Brown M, Demaerschalk BM, Hoh B, et al. 2018 Guidelines for the early management of patients with acute ischemic stroke: a guideline for healthcare professionals from the American Heart Association/American Stroke Association. Stroke. 2018 Mar; 49(3): e46-e110. doi: 10.1161/ STR.0000000000000158

3. Kamal H, Mowla A, Faroog S, Shirani P. Recurrent ischemic stroke can happen in stroke patients very early after intravenous thrombolysis. J Neuro Sci. 2015; 358: 496-497. doi: 10.1016/j.jns.2015.09.020.

4. Tanaka K, Ohara T, Ishigami A, Ikeda Y, Matsushige T, Satow T, et al. Fatal multiple systemic emboli after intravenous thrombolysis for cardioembolic stroke. J Stroke Cerebrovasc Dis. 2014 Feb; 23(2): 395-397. doi: 10.1016/j. jstrokecerebrovasdis.2013.03.007.

5. Yalcin-Cakmakli G, Akpinar E, Topcuoglu MA, Dalkara T. Right internal carotid artery occlusion during intravenous thrombolysis for left middle cerebral artery occlusion. J Stroke Cerebrovasc Dis. 2009 Jan; 18(1): 74-77. doi: 10.1016/j.jstrokecerebrovasdis.2008.08.007.

6. Oberreuter G, Silva N, Caba S, Morales M, Nieto E, Guevara C. Accidente cerebrovascular isquémico en pacientes con trombo intracavitario: experiencia con tratamientos distintos en fase aguda. Rev Med Chil. 2014 Sep; 142(9): 1200-1204. doi: 10.4067/S0034-98872014000900015.

7. Kobayashi M, Tanaka R, Yamashiro K, Ueno Y, Kato E, Miura S, et al. Preexisting mobile cardiac thrombus and the risk of early recurrent embolism after intravenous thrombolysis: a case report. J Stroke Cerebrovasc Dis. 2015 Jun; 24(6): e161-3. doi: 10.1016/j.jstrokecerebrovasdis.2015.03.001.

8. Safouris A, Kargiotis O, Magoufis G, Katsanos AH, Stamboulis E, Tsivgoulis G. Early neurological deterioration during alteplase infusion for acute ischemic stroke: an uncommon complication of intravenous thrombolysis. Neurologist. 2017 May; 22(3): 90-91. doi: 10.1097/NRL.0000000000000118. 